\title{
Interaction of $\beta$-glucan and tea during bread baking increased SCFA production in vitro
}

\author{
A. M. M. Jalil, E. Combet, C. A. Edwards and A. L. Garcia \\ Human Nutrition, School of Medicine, College of Medical, Veterinary \& Life Sciences University of Glasgow, New \\ Lister Building, Glasgow Royal Infirmary, 10-16 Alexandra Parade, Glasgow, G31 2ER, Scotland, United Kingdom
}

Tea and bread are usually consumed separately but different food matrix interactions and fermentability effects may vary when they are combined in a meal. $\beta$-glucan is a soluble dietary fibre with mixed $\beta-(1 \rightarrow 3)$ and $\beta-(1 \rightarrow 4)$ linkages; combination with tea polyphenols could form a 'physical barrier' to starch-alpha-amylase interactions. This combination will pass undigested to the large intestine, to be metabolised by the gut microbiota into short chain fatty acids (SCFA). Soluble $\beta$-glucan and tea polyphenols are metabolised by microbiota in the large intestine into $\mathrm{SCFA}^{(1,2)}$. SCFA, particularly propionate and butyrate may have key positive effects on human health ${ }^{(3)}$. Hence, the present study aimed to determine the fermentability of breads prepared with $\beta$-glucan and tea after digestion in an in vitro model of the upper gut.

Breads (white bread, WB; black tea, BT; $\beta$-glucan, BG; $\beta$-glucan plus black tea, BGT) were prepared using standard bread making techniques. Breads containing $0.5 \mathrm{~g}$ of available carbohydrate (dry weight) and wheat flour (WF) were subjected to in vitro digestion and the resulting residues were used as substrate in in vitro batch fermentation ${ }^{(4)}$. Aliquots of fermentation slurry were assayed for $\mathrm{pH}$ and SCFA (acetate, propionate and butyrate).

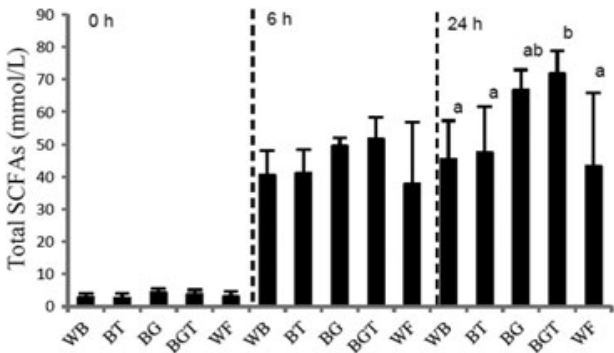

Fig. 1.

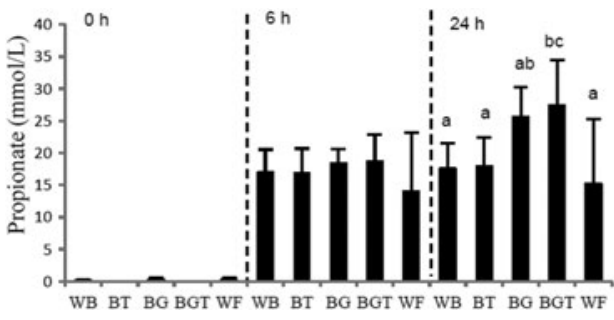

Fig. 3.

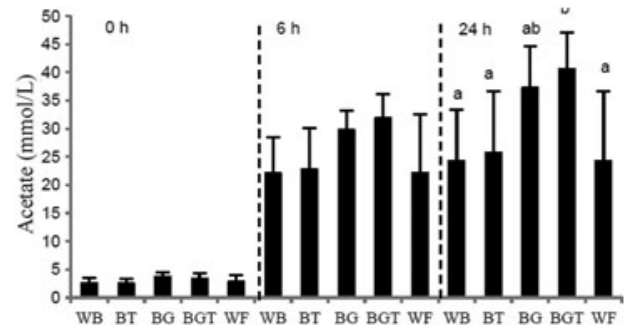

Fig. 2.

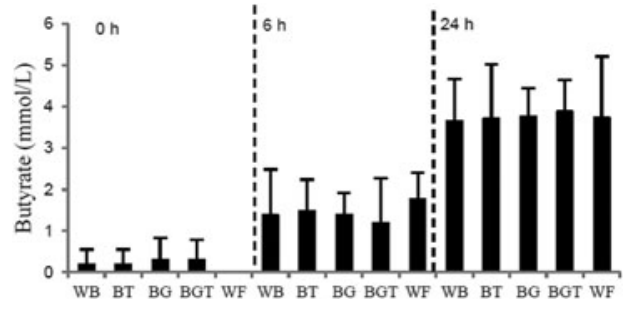

Fig. 4.

The results are expressed as mmol/L (mean, SD) of SCFA for four replicates $(n 4)$ ). Different superscripts indicate significant values within the same time point (ANOVA followed by LSD post hoc test).

All breads showed similar total SCFA at 0 and $6 \mathrm{~h}$ (Fig. 1). After $24 \mathrm{~h}, \beta$-glucan breads (BG and BGT) had significantly higher total SCFA than other breads. However, there was no effect of tea on total SCFA production. There was no difference in acetate between samples at 0 and $6 \mathrm{~h}$ (Fig. 2). $\beta$-glucan breads (BG and BGT) had significantly higher acetate than WB and BT breads. However, the addition of tea did not have any additional effects on acetate production. No significant changes were observed in propionate between samples at 0 and $6 \mathrm{~h}$ (Fig. 3). Propionate production was higher with fermented BG and BGT at $24 \mathrm{~h}$ compared with WB, BT and WF. No additional effect of tea was found on propionate. The butyrate levels were similar between samples at 0,6 and $24 \mathrm{~h}$ (Fig. 4 ). The inclusion of black tea in bread with $\beta$-glucan had no impact on SCFA production but there maybe be other effects on polyphenol metabolism but this needs further investigation.

1. Stalmach A, Troufflard S, Serafini M, et al. (2009) Mol Nutr Food Res 53 Suppl 1, S44-S53.

2. Hughes SA, Shewry PR, Gibson GR, et al. (2008) FEMS Microb Ecol 64, 482-493.

3. Arora T, Sharma R, Frost G (2011) Appetite 56, 511-515.

4. Edwards CA, Gibson G, Champ M, et al. (1996) J Sci Food Agric 71, 209-217. 